\title{
Erratum
}

\section{Detailed Balance}

\section{in Open Quantum Markoffian Systems}

\section{H.J. Carmichael and D.F. Walls}

Z. Physik B 23, 299-306 (1976)

Equation (3.25) should read

$$
\begin{aligned}
& P_{s s}^{(\Omega)}\left(\alpha, \alpha^{*}\right) \exp \left[\vec{\Lambda}^{(\Omega)}\left(\alpha, \alpha^{*}\right)\right] \overline{\mathscr{L}}^{(\Omega)}\left(\alpha, \alpha^{*}\right)^{n} \\
& =\tilde{\mathscr{L}}^{(\Omega)}\left(\alpha, \alpha^{*}\right)^{n} \tilde{P}_{s s}^{(\Omega)}\left(\alpha, \alpha^{*}\right) \exp \left[\tilde{\Lambda}^{(\Omega)}\left(\alpha, \alpha^{*}\right)\right] .
\end{aligned}
$$

Equation (3.27) should read

$P_{s s}^{(\Omega)}\left(\alpha, \alpha^{*}\right) \exp \left[\vec{\Lambda}^{(\Omega)}\left(\alpha, \alpha^{*}\right) \tau\right] \overline{\mathscr{L}}^{(\Omega)}\left(\alpha, \alpha^{*}\right)$

$=\tilde{\mathscr{L}}^{(\Omega)}\left(\alpha, \alpha^{*}\right) \tilde{P}_{s s}^{(\Omega)}\left(\alpha, \alpha^{*}\right) \exp \left[\tilde{\Lambda}^{(\Omega)}\left(\alpha, \alpha^{*}\right)\right]$.

H.J. Carmichael

D.F. Walls

Department of Physics

University of Waikato

Hamilton, New Zealand 